\title{
The Importance of Accurate Anamnesis in Determining Suspected Drugs Causing of Oral Manifestation of Stevens Johnson Syndrome-Toxic Epidermal Necrolysis (Case Report)
}

\author{
Selvia ${ }^{1}$, Indriasti I Wardhany ${ }^{2 *}$ \\ ${ }^{1 .}$ Oral Medicine Residency Program, Universitas Indonesia, Jakarta 10430, Indonesia \\ ${ }^{2}$ Oral Medicine Department, Universitas Indonesia, Jakarta 10430, Indonesia \\ *E-mail: indriasti.indah61@ @ui.ac.id
}

\begin{abstract}
Introduction: Stevens - Johnson Syndrome (SJS) and Toxic epidermal necrolysis (TEN) are drug hypersensitivity lifethreatening disease that involve skin and mucous membrane. Oral manifestation occurs in almost all the patients of SJSTEN.

Case report: A 52-years-old SJS-TEN female patient was referred from department of dermatology and venereology of RSCM hospital, Jakarta to the department of oral medicine, because of the painful ulcers in the mouth and difficulty to eat. Severe loss of integrity lesions was found in lips and oral mucosa. The causing drugs cannot be identified earlier because of challenging condition during anamnesis. The leading clue of suspected causative drugs was conducted by oral medicine specialist with in depth anamnesis of thorough patients' medical history. Allergic test after six-week period after the patient first visit shows positive result of carbamazepine and amitriptyline hypersensitivity.

Discussion: The delayed in determining causing drugs in this case were because of several causes, especially lack of information gain during anamnesis. In the condition when the operator cannot obtain sufficient information due to patient condition, it was very important to have an excellent communication in the collaborating medical team and patient family as well as constantly obtaining new important information during the ongoing treatment. Since the appropriate and successful treatment was depending in determining an exact cause, the anamnesis plays a critical role in this case.
\end{abstract}

Conclusion: Accurate anamnesis by operator was an important part regarding the successful management of patient with SJS-TEN since the disease has an enormous impact in patient's quality of life.

Keywords: amitriptyline hypersensitivity, carbamazepine hypersensitivity, Stevens-Johnson Syndrome (SJS)-Toxic Epidermal Necrolysis (TEN)

\section{Introduction}

SJS and TEN are life-threatening disease that involved skin and mucous membrane. Both are differentiated by their extent of skin detachment. ${ }^{1,2}$ SJS refers to the disease that involve body surface less than $10 \%$, and TEN refers to those with more than $30 \%$ involvement. ${ }^{1,3}$ Epidermal loss between $10 \%$ and $30 \%$ is called SJS-TEN overlap. ${ }^{1}$ Drugs are identified as the main cause of SJS-TEN cases. ${ }^{2}$ Other causes are mycoplasma pneumoniae and herpes simplex virus infections in which the etiology remains unknown. Carbamazepine is an anticonvulsant drugs which often reported induce SJS/TEN, , ${ }^{1,5}$ while amitriptyline as a tricyclic antidepressant drug also connected to severe skin symptoms. ${ }^{2,6}$ SJS/TEN frequently occurs in women and the incidence increase with age. ${ }^{1}$ Cancer, collagen vascular disease and human immunodeficiency syndrome (HIV) increase risk factor for SJS/TEN. Average mortality is $1 \%-5 \%$ for SJS and $25 \%-35 \%$ for TEN.

About $80 \%-90 \%$ of all cases of SJS/TEN involve mucous membrane include oral cavity and lips., ${ }^{2,3}$ Burning sensation, erythematous and edemas on the lips and buccal mucosa are some of the first sign in the case of SJS/TEN. Sites of the oral cavity which usually involve are labial mucosa, buccal mucosa, tongue, floor of the mouth and soft palate. Commonly there is hemorrhagic crusting on the vermilion of the lips. ${ }^{3}$ Objective of this case report is to describe one case of patient with SJS/TEN caused by drug eruption suspect carbamazepine and amitriptyline that involve lips and oral cavity. Suspected causative drugs did not known from the outset anamnesis but they were noted from excessive anamnesis 
by oral medicine specialist. Known of suspected causative drugs important to develop definitive diagnosis and obtain treatment planning. Careful and accurate anamnesis was an important part of management patient with SJS-TEN, and require good communications between dentist/ oral medicine specialist and other physicians especially dermatologist. The involvement of lips and oral cavity requires an appropriate and careful treatment, to avoid complications caused by painful oral lesions.

\section{Case Report}

A 52-years-old female patient had referred from department of dermatology and venereology RSCM Jakarta to oral medicine division with working diagnosis of drug eruption SJS-TEN type. She has been hospitalized in the last two days, complained for painful lesion on her lips and oral cavity in the past four days (two days before hospitalization), difficult to open her mouth and swallowing. The skin and perioral lesions have been moisturized with $\mathrm{NaCl} 0,9 \%$ liquid on gauze. She could not brush her teeth, but always clean her teeth and mouth with wet cotton and water. She just ate liquid diet because of soreness of the oral cavity and difficulty of swallowing. According to the initial assessment in the medical record, she had consumed some drugs include amoxicillin, FGTroches, epherisone, tramadol and vitamin C (ultra-way) for several days. From the extensive anamnesis about medical history, noted that she has been treated by neurologist for about two months because of headache and consumed some other drugs include, meloxicam, gabapentin and one type of drugs later known as carbamazepine, she also consumed amitriptyline that we know later. Thus, we share this information to the dermatologist as the main operator to follow up.

Patient did not have any history of systemic disease. No family history of the same disease or complaint, and consciousness was good. It was noted history of recurrent stomatitis 2-3 times a year. All medications before hospitalization have been discontinued.

Clinical examination, skin detachment of the body surface and peeled off with minor manipulation and appeared blackish erythematous in color. Conjunctivitis, oral mucosal lesions and lesions of genitalia was also noted. Lymph nodes of cervical, sub mandible and submental could not examined because of erosive and painful skin lesions. Perioral with erosive skin lesions, blackish erythematous in color and bullae on submental region. Hemorrhagic crusting of the lips also noted. Mid level of oral hygiene, calculi on sub and supra gingiva with erythematous gingiva. The teeth could not be examined yet. Multiple oral sloughing, erosive and erythematous of the buccal mucosa, tongue, palate, soft palate and floor of the mouth were seen. Labial mucosa could not have seen yet because of soreness. Laboratory investigation from blood examination showed decrease in leucocyte, PT and APTT, and increase rate of SGOT and SGPT.

Provisional diagnosis established oral manifestation of SJS-TEN. Treatment for oral lesions include education for patient and family specially to improve oral hygiene, instruction to do swish-and-spit $6 \mathrm{mg}$ dexamethasone disperse in $10 \mathrm{ml}$ of water in the morning and $3 \mathrm{mg}$ dexamethasone disperse in $10 \mathrm{ml}$ of water in the afternoon, moistened the lips and perioral with gauze and $\mathrm{NaCl} 0,9 \%$ solution for 3 to 5 minutes, and put the liniment of $4 \mathrm{mgs}$ dexamethasone in vaseline lanoline on the lips 3 to 4 times daily. Patient have already got treatment from dermatologist include $\mathrm{NaCl} 0,9 \%$ infusion, vaseline album as skin ointment 2 times daily, clindamycin 300mgs, Ranitidine $150 \mathrm{mgs} 2$ times daily, methyl prednisolone for injection $3 \times 4$ vial per day. Moisturize the skin with $\mathrm{NaCl} 0,9 \%$ on gauze.

On the second visit for control, four days later, patient felt better. She has already got eating soft diet, no soreness in the oral region and she could swallow normally. Patient have not been brushing her teeth because she is afraid of gingival bleeding, but always clean her teeth with gauze moistened with $\mathrm{NaCl} 0,9 \%$ due to the instruction. Limited mouth opening. The lesions on the lips and oral mucosa repaired, no complaint of new lesion. Lesions on the skin and perioral also repaired. Treatment for oral lesions continued with the same medication, but dosage of dexamethasone for swish-and-split reduce to $4 \mathrm{mgs}$ in the morning and $2 \mathrm{mgs}$ in the afternoon.

Next visit for control almost two months later, patient was referred by dermatologist to oral medicine clinic for oral lesion evaluation. Noted the suspected drugs have been identified: gabapentin, carbamazepine, meloxicam, tramadol, ketorolac, amoxicillin, epherisone $\mathrm{HCl}$, tizamidine $\mathrm{HCl}$, natrium diclofenac, vitamin $\mathrm{C}$, paracetamol and amitriptyline. Allergic test has been done when she came into the oral medicine clinic, and the results are positive for carbamazepine and amitriptyline.

Medication for oral lesions used up until 3 days after she went home from the hospital about a month ago. The entire lesions on the lips and the oral mucosa recovered fully, no complaint of pain or relapse since the last control. Intra oral examination establish moderate oral hygiene, desquamation of lips, calculus sub and supra gingiva, mobile teeth 41 and 42 regions. Treatment include oral health education, vaseline album for lips ointment and referred for scaling and periodontal treatment. 

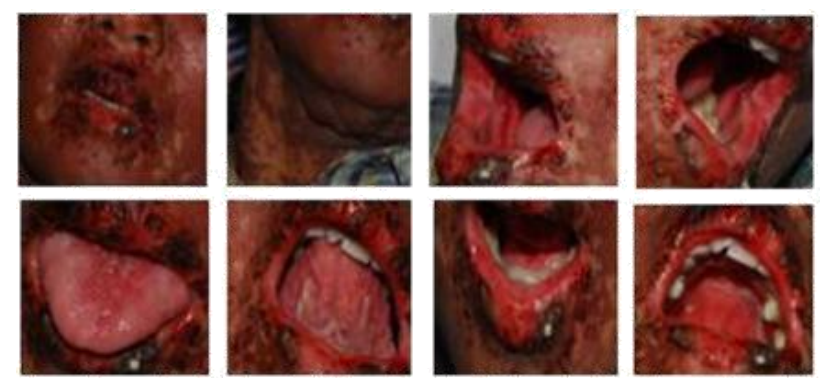

Figure 1. First visit. Clinical features of oral lesions in SJSTEN on acute phase. Involvement of all mucosal surface and crusting of the lips
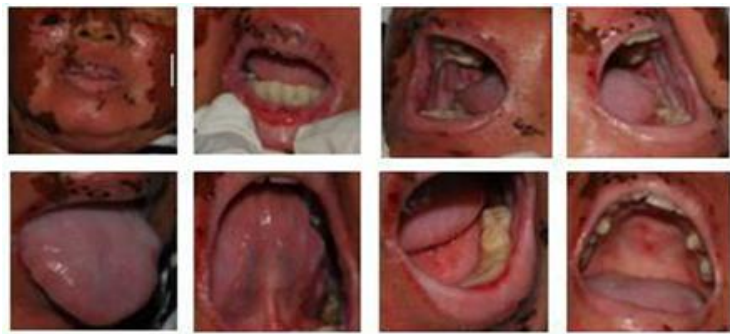

Figure 2. Second visite. Significant healing progress of oral lesions, no complaint of new lesion
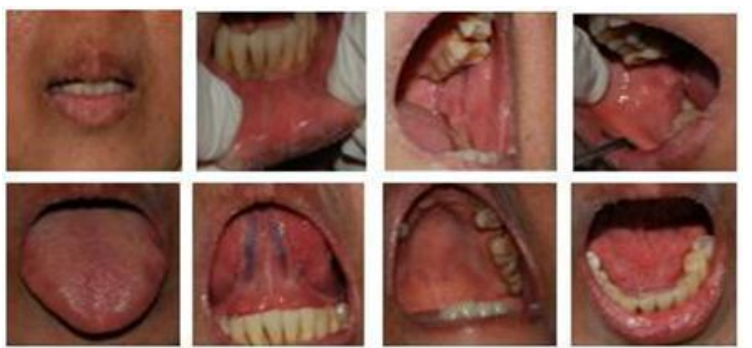

Figure 3. Two months after hospitalize. Control for oral lesion establish full healing of oral manifestation of SJSTEN

\section{Discussion}

Immune-mediated was believed as the pathogenesis of SJS-TEN. Widespread epidermal detachment that occur in patient with SJS-TEN caused by the keratinocyte apoptosis and then tissue necrosis, described in histopathologic features of SJS-TEN. The current concept of specific drug hypersensitivity reaction in SJS-TEN supported also by immunological finding besides clinical and histopathological feature. Role of cytotoxic $\mathrm{T}$ lymphocyte shows in the early phase which is observed a lot of cytotoxic CD8+ T lymphocytes in the blister fluid. Other components involve in the cytotoxicity mechanism including granzyme B, cytotoxic molecules FasL and granulysine. To date, how the culprit drug regulates the function of these key players still in ongoing researh ${ }^{2}$.

Most of the patients with SJS-TEN exhibit oral manifestation. Predilection area in the mouth could be all the oral mucosa and lips. ${ }^{2,3}$ In this case, diagnosis established due to anamnesis, clinical examination and laboratory test. Dermatologist did not perform histopathologic examination because it already clears to be SJS-TEN and they thought proper treatment could establish soon to avoid life threatening. Noted that the patient consumes some medications that may induce hypersensitivity reaction. It was an acute onset of the lesions, and exposed the predilection area of SJS-TEN, include the skin, oral mucosa, conjunctiva and genital, and might be the gastrointestinal tract. Patient did not have any history of autoimmune blistering disease. Histopathologic examination and direct immunofluorescence usually use to rule out differential diagnosis of autoimmune blistering disease. Laboratory test showed systemic condition of neutropenia, decrease of thrombocytes but still in normal value, and increase of SGOT-SGPT that support the condition of SJS-TEN. ${ }^{1,5}$

The culprit drugs could not recognize at the time of provisional diagnosis, thus for sure of the causative require the allergic test. From the patch test and prick test the causative medications recognize as carbamazepine and amitriptyline. These two drugs are anticonvulsant and antidepressant. Carbamazepine usually used as antiepileptic and antineuropatic pain, sometimes can be used for trigeminal neuralgia5. High risk to SJS/TEN occurs in the first two months. ${ }^{2}$ According to the literature, amitriptyline as a tricyclic antidepressant was one of the drugs that can induce hypersensitivity reaction. ${ }^{6}$ Patients have used these drugs for about two months when she confessed of headache and have been treating by neurologist, but we have not got more information about the treatment.

Etiology of SJS-TEN that was a hypersensitive drugs reaction, associated with genetic factor. According to the study in the Asian country include China, Thailand and Malaysia, they found there were significant association between HLA-B*1502, carbamazepine and occurrence of SJS-TEN. ${ }^{4,7,8}$ But another study in European population did not give the same result, they conclude the HLAB*1502 allele is not a universal marker for this disease and that ethnicity matter. ${ }^{9}$ Another independent cause which is well-establish to induce SJS-TEN is graft versus host disease. Infection of mycoplasma pneumonia and herpes simplex virus have been reported to induce SJSTEN in several cases. ${ }^{1,2}$

Management of SJS-TEN including treatment of acute phase, late phase and sequel. After establishing the diagnosis, the severity and prognosis should be determined. We can use SCORTEN scoring system to evaluate the prognosis in patient with SJS-TEN. The SCORTEN score in our patient determined of 2 , noted the age about 52, no malignancy and tachycardia, the initial epidermal detachment between $10 \%$ and $30 \%$. SCORTEN score of 3 or above should be managed in an intensive care unit. ${ }^{2}$. When the cause noted as $\operatorname{drug}(\mathrm{s})$, all the consumed drugs should be discontinued. This patient had stopped all the medications when she was starting the treatment in RSCM. It is important to know the chronology of administration of the consumed medications or culprit drug(s). 
Table 1. SCORTEN scoring system1,2

\begin{tabular}{|c|c|c|c|}
\hline $\begin{array}{l}\text { SCORTEN } \\
\text { Parameter }\end{array}$ & $\begin{array}{l}\text { Individual } \\
\text { Score }\end{array}$ & $\begin{array}{l}\text { SCORTEN (sum } \\
\text { of } \\
\text { scores) }\end{array}$ & $\begin{array}{l}\text { Predicted } \\
\text { mortality } \\
(\%)\end{array}$ \\
\hline $\begin{array}{l}\text { Age }>40 \\
\text { years }\end{array}$ & $\begin{array}{l}\text { Yes }=1, \\
\mathrm{No}=0\end{array}$ & $0-1$ & 3.2 \\
\hline Malignancy & $\begin{array}{l}\text { Yes }=1, \\
\text { No=0 }\end{array}$ & 2 & 12.1 \\
\hline $\begin{array}{l}\text { Tachycardia } \\
(>120 / \mathrm{min})\end{array}$ & $\begin{array}{l}\text { Yes }=1 \\
\mathrm{No}=0\end{array}$ & 3 & 35.8 \\
\hline $\begin{array}{l}\text { Initial surface } \\
\text { of epidermal } \\
\text { detachment } \\
>10 \%\end{array}$ & $\begin{array}{l}\text { Yes }=1, \\
\mathrm{No}=0\end{array}$ & 4 & 58.3 \\
\hline $\begin{array}{l}\text { Serum urea } \\
>10 \mathrm{mmcl} / 1\end{array}$ & $\begin{array}{l}\text { Yes }=1 \\
\mathrm{No}=0\end{array}$ & $>5$ & 90 \\
\hline $\begin{array}{l}\text { Serum glucose } \\
>14 \mathrm{mmcl} / 1\end{array}$ & $\begin{array}{l}\text { Yes }=1 \\
\mathrm{No}=0\end{array}$ & & \\
\hline $\begin{array}{l}\text { Bicarbonate } \\
>20 \mathrm{mmcl} / 1\end{array}$ & $\begin{array}{l}\text { Yes }=1, \\
\text { No=0 }\end{array}$ & & \\
\hline
\end{tabular}

Treatment for patient with SJS-TEN require supportive care, management of fluid and electrolyte with intravenous fluid, and transferred to the burn center could increase survival rate. ${ }^{2}$ When the patient had been referenced to us from the dermatologist, she had already got treatment with high dose systemic corticosteroid, intravenous methyl prednisolone. According to the dermatologist, these drugs had been given in a short time to avoid side effect from long term used of corticosteroid but we need the drugs as immunosuppressant for hypersensitive reaction. The role of systemic corticosteroids in SJS-TEN is controversial. ${ }^{12,10}$ The lesions may be treated with a short course of systemic glucocorticosteroid in patients without significant contraindications to their use. ${ }^{10}$ The patient was referred to oral medicine specialist to evaluate and manage the oral manifestation and noted that the oral lesions have not giving significant reaction to the systemic corticosteroid. For the lips and oral lesions, we gave topical corticosteroid. Dexamethasone for oral rinse $6 \mathrm{mg}$ disperse in $10 \mathrm{ml}$ of water in the morning and $3 \mathrm{mg}$ dexamethasone disperse in $10 \mathrm{ml}$ of water in the afternoon, and put the liniment of $4 \mathrm{mgs}$ dexamethasone in vaseline lanoline on the lips 3 to 4 times daily.

One of the interesting points of the case was related to the anamnesis. information was known from the initial assessment, and deeper anamnesis was done, the information related to the suspected causative drugs were known from extensive anamnesis of medical history by oral medicine specialist. Next, this information should be shared immediately to the dermatologist as main operator, and good communication required establishing proper diagnosis and treatment planning. Carbamazepine and amitriptyline as the causative drugs concluded later after the allergic test, but they were noted to be suspected after the extensive anamnesis of medical history. While in the case of SJS-TEN it is important to know suspected causative drugs to establish proper diagnosis, set the allergic test and avoid them from used by patient.

At the next visit for control, oral lesions evaluation established progression of healing. There was no complaint nor examination of new lesion and the lesions already existed have been improving. To avoid infection and sepsis, antibiotic clindamycin had been administered by dermatologist, the skin lesions were moistened with $\mathrm{NaCl} 0,9 \%$ on gauze as well as perioral lesions. To improve healing, skin also smeared with vaseline album ointment. Four days later, skin lesions also repaired and patient could discharge.

Two months after hospitalize, patient came to RSCM hospital to controlled and got allergic test. Dermatologist referred the patient to oral medicine specialist to evaluate oral condition and to get dental and oral treatment. When she was undergoing the allergic test, all the medications had stopped, and the treatment for dental and oral mucosa has been done without any material or medication. Allergic test showed positive reaction to carbamazepine and amitriptyline. During the time of observation after home, she had always maintained oral hygiene, brushing her teeth 2 times daily in the morning and night, so when she came for control we noticed that the oral hygiene was good.

\section{Conclusions}

One of the important parts of the management of SJSTEN is accurate and careful anamnesis to lead us knows the chronology of the disease and suspected causative agent.

Management of oral lesions in patient with SJS-TEN involve general treatment of SJS-TEN such as evaluating severity and prognosis with SCORTEN scoring system, prompt withdrawal of culprit drug(s), immediately give supportive care including intravenous fluid for management of fluid and electrolyte requirements, and systemic drug therapy. Specific treatment for oral lesions with high dose topical corticosteroid in short time for mouthwash 2 times daily and tapered and corticosteroid ointment for lips crusting showed significant effect to oral mucosal lesion and lips. Maintain good oral hygiene support the progress of oral mucosal healing.

\section{Acknowledgment}

My gratitude for Cipto Mangunkusumo Hospital for collaboration, drg. Indriasti Indah Wardhany, SpPM (Oral Medicine staff faculty of dentistry Universitas Indonesia), drg. Endah Ayu Tri Wulandari, SpPM (Oral Medicine division staff of RSCM hospital) as supervisors in evaluating the patient. Special thanks for dr. Endi Novianto, SpKK (Medical staff of Dermatology and Venereology Departement of RSCM hospital), dr. Yudo Irawan (Resident of Dermatology and Venereology Departement) as collaborating medical team. 


\section{References}

1. Ellender RP, Peters CW, Albritton HL, Garcia a J, Kaye a D. Clinical considerations for epidermal necrolysis. OchsnerJ. 2014;14(1524-5012 (Print)):413-417.

2. Harr T, French LE. Stevens-Johnson syndrome and toxic epidermal necrolysis. Chem Immunol Allergy. 2012;97:149-166.

3. Ramana BV Reddy1, P Chandra Shekar1, K Lalith Prakash Chandral RA. Oral lesions associated with Nevirapine- induced SJS-TEN.pdf. 2013.

4. Kulkantrakorn K, Tassaneeyakul W, Prabmechai Napat, et al. HLA-B * 1502 Strongly Predicts Stevens - Johnson Syndrome and Toxic Epidermal Necrolysis in Thai Patients with Neuropathic. Pain Pract. 2012;12(3):202-208.

5. Bae HM, Park YJ, Kim YH, Moon DE. Stevens-Johnson syndrome induced by carbamazepine treatment in a patient who previously had carbamazepine induced pruritus. Korean J Pain. 2013;26(1):80-83.

6. Herstowska M, Komorowska O, Cubała WJ, JakuszkowiakWojten K, Gałuszko-Wȩgielnik M, Landowski J. Severe skin complications in patients treated with antidepressants: A literature review. Postep Dermatologii i Alergol. 2014;31(2):92-97.

7. Chung WH, Hung SI, Hong HS, Hsih MS, Yang LC, Ho HC, Wu JY CY. Medical genetics: a marker for StevensJohnsontle. Syndr Nat. 2004;(428):486.

8. Chang CC, Too CL, Murad S, Hussein SH. Association of HLA-B1502 allele with carbamazepine-induced toxic epidermal necrolysis and Stevens-Johnson syndrome in the multi-ethnic Malaysian population. Int J Dermatol. 2011;50(2):221-224

9. Lonjou C, Thomas L, Borot $\mathrm{N}$, et al. A marker for \{Stevens-Johnson\} syndrome …: ethnicity matters. Pharmacogenomics J. 2006;6(4):265-268.

10. Mehdipour M, Zenouz AT. Role of Corticosteroids in Oral Lesions;2007. 